\title{
Speciation and amphotericin B sensitivity studies on blood isolates of Candida from burned patients
}

\author{
DONALD D. STIERITZ, EDWARD J. LAW, AND IAN ALAN HOLDER \\ From the University of Cincinnati College of Medicine, the Departments of Microbiology and Surgery, \\ and Shriners Burns Institute, Cincinnati Unit, Cincinnati, Ohio
}

SYNOPSIS Methods of speciating Candida isolates from clinical specimens are described and the necessity of speciation is emphasized. Differences in susceptibility of $C$. albicans and $C$. tropicalis to amphotericin B were observed and the implications of this in relation to treatment with amphotericin $B$ and the development of resistance are discussed.

In recent years, the problem of infection in burns has become increasingly complicated with the increased incidence and recognition of yeasts and other fungi as major aetiological agents of burn wound sepsis (Bruck, Nash, Foley, and Pruitt, 1971; Nash, Foley, and Pruitt, 1970; MacMillan, Law, and Holder, 1972). The seriousness of the problem is accentuated by the limited and often toxic antibiotic therapy available for treatment of systemic mycoses.

A study at the Cincinnati Unit of the Shriners Burns Institute showed $5 \cdot 1 \%$ of 271 burned patients at some time in the course of their stay in hospital had positive blood cultures for Candida. Within a five-year period, 11 of 75 deaths were documented on postmortem examination to be caused by disseminated candidiasis (MacMillan et al, 1972).

Factors predisposing to candidiasis have been reviewed extensively (Seelig, 1966; Hart, Russell, and Remington, 1969; Taschdjian, Kozinn, and Toni, 1970). The organism is generally classified as a member of the normal flora, with its pathological potentiality coming into play only when a patient suffers from an underlying disease or is subjected to treatment regimens compromising normal hostdefence mechanisms.

While Candida albicans is considered the primary agent of this disease, $C$. tropicalis has also been shown to produce severe systemic infections (Conn, Crean, and Maccabe, 1959; Richart and Dammin, 1960; Hurley and Winner, 1962; Louria, Blevins, Armstrong, Burdick, and Lieberman, 1967).

Diagnosis of candidiasis depends primarily on the demonstration of the fungus in the blood and tissues. The clinical manifestations are not always specific, and attempts to culture the organism from the blood often fail (Goldstein and Hoeprich, 1972).

Received for publication 3 April 1973.
During a two-year period at this Institute, 12 patients sustaining major thermal injury ( $>30 \%$ total body surface) had cultures of blood and intravenous catheter tips positive for Candida. A review of patients with positive blood cultures showed that a majority of the cultures were reported as Candida species rather than $C$. albicans. In addition, there were seven deaths in this group despite the initiation of parenteral therapy with amphotericin B.

Subsequent to this review, a retrospective laboratory study was conducted to determine accurately the species of these isolates and their susceptibility in vitro to amphotericin $\mathrm{B}$.

\section{Materials and Methods}

\section{PATIENT STUDY GROUP}

The study includes 12 patients treated at this Institute during the years $1969-1970$ for major thermal burns in excess of $30 \%$ total body surface. These patients were diagnosed as having candidaemia on the basis of multiple positive blood cultures.

\section{CANDIDA ISOLATES FROM BLOOD AND \\ INTRAVENOUS CATHETERS}

A total of 89 cultures originally isolated from this group of patients was stored in a desiccated state at $5^{\circ} \mathrm{C}$. Attempts to revitalize them 12-18 months later resulted in the recovery of 50 viable cultures. At least one representative culture was recovered for each of the 12 patients. Cultures were checked for purity on sheep blood agar plates and transferred and maintained on Sabouraud dextrose agar slants.

\section{SPECIATION OF CANDIDA}

Cultures were inoculated onto cornmeal agar slants 405 
and incubated at $25^{\circ} \mathrm{C}$ for up to 14 days. Periodic microscopic examinations were made to observe formation of chlamydospores. Chlamydospore formation is generally associated with $C$. albicans (Bailey and Scott, 1970).

The production of germ tubes was determined by the inoculation of $0.25 \mathrm{ml}$ of pooled normal human serum with each isolate and incubation at $35^{\circ} \mathrm{C}$ for two to four hours. The presence of microscopically visible germ tubes within this time is indicative of $C$. albicans (Mackenzie, 1962).

Carbohydrate assimilation tests were performed according to the method of Martin and Schneidau (1970). Agar slants of yeast nitrogen base with a $1 \%$ final concentration of carbohydrate were inoculated with the isolates. All slants were incubated at $25^{\circ} \mathrm{C}$ for up to 14 days, and positive results were indicated by the observation of growth in excess of that seen on control slant (without carbohydrate). Sugars tested are listed in table I.

Carbohydrate fermentation tests were performed in beef extract broth with bromothynol blue indicator and $1 \%$ final concentration of the respective sugars (table I). Before inoculation the cultures were serially transferred on beef extract agar slants (without carbohydrate). Following inoculation, all tubes were overlaid with $2 \mathrm{~cm}$ of sterile mineral oil and incubated at $35^{\circ} \mathrm{C}$ for up to 14 days.

\section{AMPHOTERICIN B SUSCEPTIBILITY TESTS}

The susceptibility in vitro of Candida isolates to the antifungal drug amphotericin B (Fungizone-E. R. Squibb \& Sons, Inc) was determined by a tubedilution procedure.

Aliquots of a stock solution $(100 \mu \mathrm{g} / \mathrm{ml})$ of amphotericin $\mathrm{B}$, stored at $-70^{\circ} \mathrm{C}$, were thawed and immediately diluted twofold to a final concentration of $0.02 \mu \mathrm{g} / \mathrm{ml}$. All dilutions were made in antibiotic medium no. 1 (Difco) containing $1 \%$ glucose. Because of the light-sensitive nature of solutions of amphotericin B, an initial MIC of a test isolate of
C. albicans was established with the freshly prepared stock solution. When this assay was repeated with the frozen aliquots of the stock solution, no activity was lost under our storage conditions.

The test organism was grown in brain heart infusion broth at $35^{\circ} \mathrm{C}$ for 18 to 20 hours and was diluted so that a final concentration of cells added to the antifungal assay tubes was approximately $5 \times 10^{5} / \mathrm{ml}$.

The endpoint or minimal inhibitory concentration (MIC) was recorded as the lowest concentration of the drug that prevented growth of the test organism following incubation at $35^{\circ} \mathrm{C}$ for 48 hours.

\section{Results}

The expected results of speciation tests for Candida, including the sugars used for fermentation and assimilation tests, are listed in table I. Based on these criteria, it was determined that 15 isolates recovered from five patients were $C$. albicans, while 35 cultures from seven patients were $C$. tropicalis. No other species of Candida were encountered among the isolates tested. Multiple isolates from a given patient were consistently of the same species. In one of the patients, a total of 16 cultures obtained over a sixweek interval all proved to be Candida tropicalis. Within the total group of patients, a single isolate for an intravenous catheter was identified as $C$. albicans while blood culture isolates from this same patient were $C$. tropicalis.

Table II lists the results of amphotericin B sensitivity tests of the Candida isolates. The range of MICs for $C$. tropicalis isolates was from 0.08 to 0.64 $\mu \mathrm{g} / \mathrm{ml}$ while the range of MICs for $C$. albicans isolates was from 0.08 to $0.16 \mu \mathrm{g} / \mathrm{ml}$. Six of the seven patients with $C$. tropicalis had isolates with MICs which were higher than those obtained for any of the C. albicans. The ranges of values shown for some of the patients were not necessarily sequential. In fact, most showed a random variation in the MIC versus

\begin{tabular}{|c|c|c|c|c|c|c|c|c|}
\hline & & \multicolumn{7}{|c|}{ Candida Isolates } \\
\hline & & albicans & tropicalis & $\begin{array}{l}\text { pseudo- } \\
\text { tropicalis }\end{array}$ & stellatoidea & krusei & parakrusei & guilliermondi \\
\hline \multirow{2}{*}{\multicolumn{2}{|c|}{$\begin{array}{l}\text { Chlamydospore production } \\
\text { Germ tube production }\end{array}$}} & + & $-( \pm)$ & - & \pm & - & - & - \\
\hline & & + & - & - & \pm & - & - & - \\
\hline \multirow{6}{*}{ Fermentation } & (Dextrose & AG & AG & AG & $\overline{\mathbf{A G}}$ & $\mathbf{A G}$ & $\mathbf{A G}$ & $\mathbf{A G}$ \\
\hline & Maltose & $\mathbf{A G}$ & AG & - & $\mathbf{A G}$ & - & - & - \\
\hline & Sucrose & A/- & $\mathbf{A} / \mathbf{A G}$ & AG & - & - & $\mathbf{A} /-$ & $\mathbf{A G}$ \\
\hline & Lactose & - & - & AG & - & - & - & - \\
\hline & $\int$ Dextrose & + & + & + & + & + & + & + \\
\hline & Maltose & + & + & - & + & - & + & + \\
\hline \multirow[t]{3}{*}{ Assimilation } & Sucrose & + & + & + & - & - & + & + \\
\hline & Cellobiose & - & $+( \pm)$ & + & - & - & - & + \\
\hline & Raffinose & - & - & + & - & - & - & + \\
\hline
\end{tabular}

Table I Expected results of speciation tests used in the identification of Candida isolates 


\begin{tabular}{lllll}
\hline Organism & $\begin{array}{l}\text { Patient } \\
\text { No. }\end{array}$ & $\begin{array}{l}\text { MIC Range } \\
(\mu \mathrm{g} / \mathrm{ml})\end{array}$ & $\begin{array}{l}\text { No. of } \\
\text { Cultures }\end{array}$ & $\begin{array}{l}\text { Amphotericin } \\
\text { Treatment }\end{array}$ \\
\hline C. tropicalis & 1 & $0.08-0.32$ & 16 & + \\
& 2 & $0.08-0.64$ & 8 & - \\
& 3 & $0.08-0 \cdot 32$ & 6 & - \\
& 4 & $0 \cdot 16-0.64$ & 2 & + \\
& 5 & $0 \cdot 08$ & 1 & - \\
& 6 & $0 \cdot 32$ & 1 & - \\
C. albicans & 7 & $0 \cdot 32$ & 1 & - \\
& 1 & $0 \cdot 08-0 \cdot 16$ & 9 & + \\
& 2 & $0 \cdot 08-0 \cdot 16$ & 2 & - \\
& 3 & 0.08 & $1^{1}$ & - \\
& 4 & 0.08 & $1^{1}$ & - \\
& 5 & $0 \cdot 16$ & 1 & - \\
& 6 & $0 \cdot 16$ & 1 & - \\
\hline
\end{tabular}

Table II Susceptibility of Candida isolates to amphotericin B

${ }^{1}$ From intravenous catheters

the time of obtaining the culture. There was no evidence of development of resistance to amphotericin B in Candida isolates from any of the patients who were treated with this drug. The range of MICs was essentially the same as for isolates obtained from those not receiving the drug.

An analysis of the patients in this study separated on the basis of the infecting organism is shown in tables III and IV. Before the emergence of candidaemia all 12 patients were treated with topical antibiotic agents (gentamicin five, mafenide four, silver sulphadiazine three), and all but three received prior systemic antimicrobial agents. There was no

\begin{tabular}{lllll}
\hline Patient & Amphotericin $\begin{array}{l}\text { Blood } \\
\text { Clearance }\end{array}$ & Death & $\begin{array}{l}\text { Candida at } \\
\text { Necropsy }\end{array}$ \\
\hline 1 & $+(13)^{1}$ & + & + & + \\
2 & $+(6)^{1}$ & + & + & + \\
3 & $+(12)^{1}$ & + & - & \\
4 & - & + & - & \\
5 & - & + & - & \\
\hline
\end{tabular}

Table III Summary of data from patients infected with C. albicans

${ }^{1}$ No. of days treated

\begin{tabular}{|c|c|c|c|c|}
\hline Patient & Amphotericin & $\begin{array}{l}\text { Blood } \\
\text { Clearance }\end{array}$ & Death & $\begin{array}{l}\text { Candida at } \\
\text { Necropsy }\end{array}$ \\
\hline $\begin{array}{l}1 \\
2 \\
3 \\
3 \\
4 \\
5 \\
6 \\
7\end{array}$ & $\begin{array}{l}+(11)^{1} \\
+(8)^{1} \\
+(3)^{1} \\
- \\
- \\
-\end{array}$ & $\begin{array}{l}- \\
+ \\
+ \\
- \\
- \\
+ \\
+\end{array}$ & $\begin{array}{l}+ \\
+ \\
+ \\
+ \\
+ \\
+ \\
-\end{array}$ & $\begin{array}{l}+ \\
+ \\
+ \\
+ \\
+\end{array}$ \\
\hline
\end{tabular}

Table IV Summary of data from patients infected with C. tropicalis

'No. of days treated correlation between these treatments and the resulting Candida species recovered from the blood.

Before the candidaemia, routine cultures of the burn wound surface and the urine were positive for Candida in all 12 patients. Stool cultures were positive in eight. Recovery of Candida from these other sites preceded the candidaemia from one to 14 days and the species of these isolates was generally of that subsequently recovered from the blood.

In the $C$. albicans group (table III) three patients were treated with amphotericin B and subsequent blood cultures indicated the clearance of the organism from the blood in all three. However, two of these patients eventually died and postmortem examination demonstrated the presence of disseminated infection by Candida in both.

The $C$. tropicalis group (table IV) also had three patients treated with amphotericin $B$, but in one blood cultures remained positive until death, despite administration of the drug. These three patients as well as two others who received no specific systemic antifungal therapy subsequently died and histological and bacteriological postmortem studies demonstrated the dissemination of Candida in all five.

\section{Discussion}

It has been the impression of clinicians that patients infected by Candida other than C. albicans were not in life-threatening situations and, therefore, less concern was given to these infections. Our data and those of others (Louria et al, 1967) indicate that $C$. tropicalis and perhaps other Candida species cause severe and fatal infections. From a clinical point of view, this means that all Candida isolates fion patients should be speciated with the tests which are readily available and easy to perform. A batter $y$ of tests, such as described here, should te used to identify each isolate precisely, rather than a few tests which only separate $C$. albicans from the other Candida organisms but do not provide information as to which species other than $C$. albicans have been isolated. Also, in using only a few differential tests, even $C$. albicans may be misidentified. This is evidenced by virtue of the fact that four of our isolates which were, before this study, identified as C. albicans turned out to be $C$. tropicalis when a battery of differential tests was used. This becomes more important since new antifungal drugs that are available may be more appropriately used with $C$. tropicalis and other Candida, which may require higher serum levels of the toxic drug amphotericin B than does $C$. albicans.

Studies on the sensitivity in vitro of clinical isolates of Candida species to amphotericin B as well as the responsiveness of the treated patients indicate that $C$. 
tropicalis may be less amenable to therapy with this drug.

A recent report by Athar and Winner (1971) indicates that clinical isolates of $C$. tropicalis are more resistant in vitro to polyene antibiotics than is C. albicans. However, they found all isolates of Candida to be sensitive to amphotericin B and nystatin on initial testing.

In our study the MIC values for $C$. tropicalis isolates were generally higher than those obtained for C. albicans. Fourteen $(40 \%)$ of the 35 cultures of $C$. tropicalis tested had a MIC in excess of $0.16 \mu \mathrm{g} / \mathrm{ml}$ which was the highest recorded with any of the $C$. albicans isolates.

The patients in the study treated with amphotericin $B$ received a maximum dosage of $1.0 \mathrm{mg} / \mathrm{kg} /$ day following an initial dosage of $0.5 \mathrm{mg} / \mathrm{kg} /$ day. While serum from these patients was not available for determination of levels of the antibiotic during therapy, the MIC values of all of the isolates are within the range of usual serum concentration attainable when given acceptable doses of this drug. Published figures indicate this level to be about $1.5 \mu \mathrm{g} / \mathrm{ml}$ (Bindschadler and Bennett, 1969; Fields, Bates, and Abernathy, 1970, 1971).

We did not find any correlation between an increase in MIC needed to inhibit the growth of Candida when compared to the number of days that any of the treated patients were given amphotericin B. This implies that development of resistance to amphotericin B does not commonly develop in vivo or if it does it requires treatment for more than 13 days. There have been reports of development of resistance to amphotericin B in vitro (Littman, Pisano, and Lancaster, 1958; Athar and Winner, 1971).

In six patients, three with $C$. albicans and three with $C$. tropicalis sepsis, who were treated with amphotericin B, only one survived. All of these patients had sustained large burns with all the concomitant problems that that implies, and factors other than Candida sepsis were present which may have contributed to their death. In four of the patients who died, treatment with amphotericin B did clear the yeast from the blood, but Candida was found in the tissues on postmortem examination. Therefore, clearance from blood after treatment with amphotericin $B$ should not be viewed as a positive prognostic sign.
Data on serum concentrations of the drug in this study are lacking and current studies at this Institute are aimed at determining these data and to correlate them with clinical responsiveness.

We would like to thank E. R. Squibb and Sons, Inc, for financial support for this project, and Robert L. Vann, MD, for his cooperation.

\section{References}

Athar, M. A., and Winner, H. I. (1971). The development of resistance by Candida species to polyene antibiotics in vitro. J. med. Microbiol., 4, 505-517.

Bailey, W. R., and Scott, E. G. (1970). Laboratory diagnosis of mycotic infections. In Diagnostic Microbiology, 3rd ed., pp. 266-269. St. Louis.

Bindschadler, D. D., and Bennett, J. E. (1969). A pharmacologic guide to the clinical use of amphotericin B. J. infect Dis., 120, 427-436.

Bruck, H. M., Nash, G., Foley, F. D., and Pruitt, B. A., Jr. (1971). Opportunistic fungal infection of the burn wound with Phycomycetes and Aspergillus. Arch. Surg., 102, 476-482.

Conn, N. K., Crean, G. P., and Maccabe, A. F. (1959). Systemic candidiasis and endocarditis due to C.tropicalis. Brit. med.J., 1, 944-947.

Fields, B. T., Jr., Bates, J. H., and Abernathy, R. S. (1970). Amphoteric in B serum concentrations during therapy. Appl. Microbiol., 19, 955-959.

Fields, B. T., Jr., Bates, J. H., and Abernathy, R. S. (1971). Effect of rapid intravenous infusion on serum concentrations of amphotericin B. Appl. Microbiol., 22, 615-617.

Goldstein, E., and Hoeprich, P. D. (1972). Problems in the diagnosis and treatment of systemic candidiasis. J. infec. Dis., 125, 190193.

Hart, P. D., Russell, E., Jr., and Remington, J. S. (1969). The compromised host and infection. II. Deep fungal infection. $J$. infect. Dis., 120, 169-191.

Hurley, R., and Winner, H. I. (1962). The pathogenicity of Candida tropicalis. J. Path. Bact., 84, 33-38.

Littman, M. L., Pisano, M. A., and Lancaster, R. M. (1958). Induced resistance of Candida species to nystatin and amphotericin $B$. Antibiot. Ann., 981-987.

Louria, D. B., Blevins, A., Armstrong, D., Burdick, R., and Lieberman, P. (1967). Fungemia caused by 'nonpathogenic' yeasts. Arch. intern. Med., 119, 247-252.

Mackenzie, D. W. R. (1962). Serum tube identification of Candida albicans. J. clin. Path., 15, 563-565.

MacMillan, B. G., Law, E. J., and Holder, I. A. (1972). Experience with Candida infections in the burn patient. Arch. Surg., 104, 509-514.

Martin, M. V., and Schneidau, J. D. Jr., (1970). A simple and reliable assimilation test for the identification of Candida species. Amer. J. clin. Path., 53, 875-879.

Nash, G., Foley, F. D., and Pruitt, B. A., Jr. (1970). Candida burn wound invasion. Arch. Path., 90, 75-78.

Richart, R., and Dammin, G. J. (1960). Candida tropicalis as a pathogen for man. New Engl. J. Med., 263, 474-477.

Seelig, M. S. (1966). The role of antibiotics in the pathogenesis of Candida infections. Amer. J. Med., 40, 887-917.

Taschdjian, C. L., Kozinn, P. J., and Toni, E. F. (1970). Opportunistic yeast infections, with special reference to candidiasis. Ann. N.Y. Acad. Sci., 174, 606-622. 